\title{
Approximate Minimum BER Power Allocation for MIMO-THP System
}

\author{
Xiang Yun, Tiejun Lv, Member, IEEE, Ping Gong, Xinghui Su and Shaoshi Yang \\ Department of Information Engineering \\ Beijing University of Posts and Telecommunications (BUPT) \\ Beijing, China \\ yunxiang_bupt@yahoo.cn, lvtiejun@tsinghua.org.cn
}

\begin{abstract}
This paper proposes a transmit power allocation (TPA) scheme based on multiple-input multiple-output (MIMO) Tomlinson-Harashima precoding (THP) structure, where a TPA matrix is introduced to the conventional MIMO-THP. We analyze the influence of the introduced TPA matrix on the performance of MIMO-THP. The proposed TPA scheme invokes the minimum average uncoded bit-error rate (BER) criterion subjected to a sum-power constraint. During the derivation, we consider the effects of precoding loss factor on the TPA scheme and obtain a closed-form expression of the TPA. Compared to existing TPA methods for MIMO-THP systems, the proposed scheme reduces processing complexity and improves the BER performance.
\end{abstract}

Keywords- multiple-input multiple-output (MIMO);TomlinsonHarashima precoding (THP); Minimum bit-error rate (MBER); transmit power allocation (TPA).

\section{INTRODUCTION}

Nowadays, multiple-input multiple-output (MIMO) system has been considered as one of the most important techniques owing to the significantly higher capacity improvement than single-input single-output (SISO) wireless channel [1], [2].The Vertical Bell Laboratories Layered Space-Time (V-BLAST) system, originally proposed in [3], is one of the most prominent schemes to achieve this higher spectral efficiency. Ginis and Cioffi [4] proved that V-BLAST receiver processing is equivalent to the MIMO Decision Feedback Equalization (DFE) structure.

Above methods require channel state information (CSI) only at the receiver side. In some cases, if the communication environment is slowly time varying, the CSI at transmitter is possible obtained by feedback in frequency-division duplex (FDD) systems or the reciprocal principle in time-division duplex (TDD) systems. Fischer et al. [5] first introduce the Tomlinson-Harashima precoding (THP) structure to eliminate the spatial interference in MIMO channel. This scheme moves the feedback filters of MIMO-DFE to the transmitter to avoid the error propagation, which is similar to the original THP used in dispersive SISO channel [6], [7]. Because the feed-forward filters are still at the receivers, it should be pointed out that the received signals of the method [5] must be cooperatively processed. Modulo operators in MIMO-THP systems result in a transmit power increase, which is quantified by precoding loss [5], [8], [11].

In order to make full use of CSI, transmit power allocation (TPA) schemes can achieve a performance improvement for MIMO systems. From the point view of maximizing channel capacity, TPA strategies were derived in [9], [10] for $\mathrm{V}$ BLAST systems and [11], [12] for MIMO-THP systems. Alternatively, from the point view of minimizing error rate performance, TPA strategies were derived in [13], [14] for VBLAST systems and [15] for MIMO-THP systems. The scheme in [15], which approximately minimizes the average Symbol-Error Rate (SER) under sum-power constraint, achieves better performance than MIMO-THP systems without TPA. However, the existing scheme needs iterative procedure and does not consider the precoding loss which is not negligible at lower constellations.

In this paper, we bring a TPA matrix into the conventional MIMO-THP system and analyze the modification of MIMOTHP structure employed with TPA scheme first. Then an effective TPA method that minimizes the bit-error rate (BER) performance under sum-power constraint is proposed. This method is capable of achieving further performance improvement because the precoding loss factor is considered in the derivations. When employed to uncoded QAM, a tighter approximation expression to the complementary cumulative distribution function (Q-function) is introduced. Finally, a closed-form expression of TPA scheme is derived.

The rest of this paper is organized as follows. The modified MIMO-THP systems for TPA are described in section II. In section III, the proposed TPA scheme is derived for this modified MIMO-THP structure in perfect CSI case. Simulation results are given in Section IV and conclusions are followed in Section V.

\section{MODIFIED MIMO-THP SYSTEM}

\section{A. System Model and Assumptions}

In this paper, we assume the transmitter has perfect knowledge of the CSI and consider the MIMO system equipped with $N_{T}$ transmit antennas and $N_{R}$ receive antennas. For simplicity, we assume both sides of the system have equal

This research has been supported by the Program for New Century Excellent Talents in University of China. 


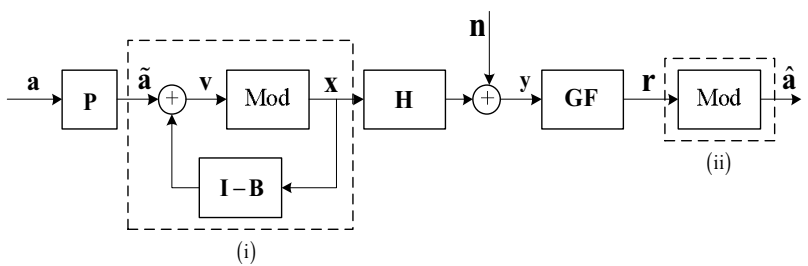

Figure 1. Block diagram of Modified MIMO-THP for TPA.

antennas number (i.e. $N_{T}=N_{R}=K$ ). Let $\mathbf{x}=\left[x_{1}, \ldots, x_{K}\right]^{T}$ and $\mathbf{y}=\left[y_{1}, \ldots, y_{K}\right]^{T}$ represent the transmitted and the received signals, respectively. The system model can be written as

$$
\mathbf{y}=\mathbf{H x}+\mathbf{n} \text {. }
$$

$\mathbf{H}=\left[h_{i j}\right]$ is the $K \times K$ channel gain matrix. The channel gain from transmit antenna $j$ to receive antenna $i$ is denoted by $h_{i j}$ and assumed to be normalized i.i.d. zero-mean complex Gaussian random variables(i.e. flat fading case). $\mathbf{n}=\left[n_{1}, \ldots, n_{K}\right]^{T}$ denotes the channel noise, which is uncorrelated white Gaussian noise with noise power $\sigma_{n}^{2}$ per dimension (i.e. $\mathbf{R}_{\mathbf{n}}=\mathrm{E}\left[\mathbf{n} \mathbf{n}^{H}\right]=\sigma_{n}^{2} \mathbf{I}$ ). It is assumed that the transmitter knows the noise variance $\sigma_{n}^{2}$.

The definitions of some symbols and operators used in this paper are listed below.

a : Boldface small symbol represents a vector

H : Boldface capital symbol represents a matrix

$\operatorname{diag}\left[x_{1}, x_{2}, \ldots, x_{D}\right]:$ A diagonal matrix with diagonal entries $x_{1}, x_{2}, \ldots, x_{D}$

$\operatorname{diag}(\mathbf{A})$ : A vector made by the diagonal entries of $\mathbf{A}$

$\left[a_{i j}\right]$ : The entry at the $i$ th row and $j$ th column of $\mathbf{A}$

$\operatorname{tr}(\cdot)$ : Trace of a matrix

$\mathrm{E}(\cdot)$ : Expectation

$(\cdot)^{T}:$ Transpose

$(\cdot)^{H}$ : Conjugate transpose

\section{B. Modified THP Structure}

We first review the MIMO-THP scheme presented in [5] with some modifications. The overall system structure is illustrated as a block diagram in Fig. 1, where the TPA matrix $\mathbf{P}$ is introduced to the structure in [5]. Here $\mathbf{I}$ is identity matrix, $\mathbf{B}$ is unit lower triangular (lower left triangular matrix with ones on the main diagonal), $\mathbf{F}$ is unitary, $\mathbf{G}$ and $\mathbf{P}$ are diagonal matrices. $\mathbf{a}=\left[a_{1}, \ldots, a_{K}\right]^{T}$ is the data vector. Each $a_{k}, k=1, \ldots, K$ uses the same $\mathrm{M}$-ary square constellation ( $M$ is

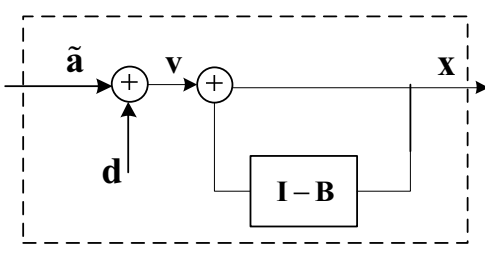

(i)

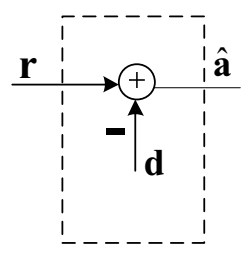

(ii)
Figure 2. Alternative linear representations of modulo operators in Fig. 1.

squared number) and has unity power (i.e. $\sigma_{a_{k}}^{2}=\sigma_{a}^{2}=1, \forall k$ ). $\mathbf{P}=\operatorname{diag}\left[\sqrt{P_{1}}, \ldots, \sqrt{P_{K}}\right]$ is the TPA matrix. $\tilde{\mathbf{a}}=\mathbf{P a}$ is the power loaded data vector and the variance of each component is $\sigma_{\tilde{a}_{k}}^{2}=P_{k} \cdot \sigma_{a_{k}}^{2}=P_{k}, k=1, \ldots, K$. The feedback matrix $\mathbf{B}$ and feed-forward matrix $\mathbf{G F}$ can be calculated under $\mathrm{ZF}$ or MMSE criteria. If we use ZF criterion, the filter matrices $\mathbf{G}$, $\mathbf{B}$ and $\mathbf{F}$ can be obtained by performing QL-factorization to H [5], i.e.

$$
\begin{aligned}
& \mathbf{H}=\mathbf{Q S}, \\
& \mathbf{G}=\operatorname{diag}\left[s_{11}^{-1}, \ldots, s_{K K}^{-1}\right], \\
& \mathbf{B}=\mathbf{G S}, \\
& \mathbf{F}=\mathbf{Q}^{-1}=\mathbf{Q}^{H} .
\end{aligned}
$$

$\mathbf{S}=\left[s_{i j}\right]$ is a lower triangular matrix, $\mathbf{Q}$ is a unitary matrix. In this case the covariance matrix of noise $\tilde{\mathbf{n}}=\mathbf{G F n}$ is

$$
\begin{aligned}
& \mathbf{R}_{\tilde{\mathbf{n}}}=\mathrm{E}\left[\tilde{\mathbf{n}} \tilde{\mathbf{n}}^{H}\right]=\sigma_{n}^{2} \mathbf{G} \mathbf{G}^{H} \\
& =\operatorname{diag}\left[\sigma_{n}^{2} / s_{11}^{2}, \ldots, \sigma_{n}^{2} / s_{K K}^{2}\right]
\end{aligned}
$$

On an alternative criterion, the MMSE based solution has also been given in [5]. The error is defined as

$$
\mathbf{e}=\mathbf{r}-\mathbf{v}=\mathbf{G F y}-\mathbf{B x},
$$

and the covariance matrix of error is $\mathbf{R}_{\mathbf{e}}=\mathrm{E}\left[\mathbf{e e}^{H}\right] . \mathrm{By}$ minimizing the mean-square error $\operatorname{tr}\left(\mathbf{R}_{\mathbf{e}}\right)$, we perform a (modified) Cholesky factorization of $\mathbf{H}^{H} \mathbf{H}$ to obtain the filter matrices, i.e.

$$
\begin{aligned}
& \mathbf{H}^{H} \mathbf{H}+\boldsymbol{\zeta} \mathbf{I}=\mathbf{S}^{\prime H} \mathbf{S}^{\prime}, \\
& \mathbf{G}^{\prime}=\operatorname{diag}\left[s_{11}^{\prime-1}, \ldots, s_{K K}^{\prime-1}\right], \\
& \mathbf{B}^{\prime}=\mathbf{G}^{\prime} \mathbf{S}^{\prime}, \\
& \mathbf{F}^{\prime}=\mathbf{S}^{\prime-H} \mathbf{H}^{H},
\end{aligned}
$$

where $\varsigma=\sigma_{n}^{2} / \sigma_{a}^{2}=\sigma_{n}^{2}$ and $\mathbf{S}^{\prime}=\left[s_{i j}^{\prime}\right]$ is a lower triangular matrix. The covariance matrix of the error vector $\mathbf{e}$ is

$$
\begin{aligned}
& \mathbf{R}_{\mathbf{e}}=\mathrm{E}\left[\mathbf{e e}^{H}\right]=\operatorname{diag}\left[\sigma_{e_{1}}^{2}, \ldots, \sigma_{e_{K}}^{2}\right] . \\
& =\operatorname{diag}\left[\sigma_{n}^{2} / s_{11}^{\prime 2}, \ldots, \sigma_{n}^{2} / s_{K K}^{\prime 2}\right]
\end{aligned}
$$


From (3) and (6), we can see that the structures of noise and error covariance matrices are similar. Thus, the transmit power can be calculated as the same manner both in ZF and MMSE cases.

\section{Equivalent Sub-channel Model}

By considering Fig.1, Fig.2 and properties of THP matrices, the detected signal vector $\hat{\mathbf{a}}$ is given as

$$
\hat{\mathbf{a}}=\operatorname{Mod}_{\mathbf{t}}\left(\tilde{\mathbf{a}}+\mathbf{d}+\mathbf{I}^{\prime}+\tilde{\mathbf{n}}\right),
$$

and can be decoupled into $K$ parallel streams [15]

$$
\hat{a}_{k}=\operatorname{Mod}_{t_{k}}\left(\tilde{a}_{k}+d_{k}+I_{k}^{\prime}+\tilde{n}_{k}\right), \forall k,
$$

where $\mathbf{I}^{\prime}=\left[I_{1}^{\prime}, \ldots, I_{K}^{\prime}\right]^{T}$ is the residual spatial Co-Channel Interference(CCI) after MMSE criterion on THP filters (for ZF case $\mathbf{I}^{\prime}=\mathbf{0}$ ), The modulo operator is introduced to reduce the transmit signal power, where $\mathbf{t}=\left[t_{1}, \ldots, t_{K}\right]$ is the modulo bound vector. $\operatorname{Mod}_{t_{k}}(\bullet)$ is modulo $t_{k}$ operator to bound its output on $T_{k}=\left[-t_{k} / 2, t_{k} / 2\right)+j \cdot\left[-t_{k} / 2, t_{k} / 2\right), \forall k$ and is defined for a complex variable $c$ as

$$
\operatorname{Mod}_{t_{k}}(c) \triangleq c-\left\lfloor\frac{\operatorname{Re}(c)}{t_{k}}+\frac{1}{2}\right\rfloor t_{k}-j\left\lfloor\frac{\operatorname{Im}(c)}{t_{k}}+\frac{1}{2}\right\rfloor t_{k},
$$

where the floor operator $\lfloor\cdot\rfloor$ rounds the argument to the nearest integer towards minus infinity. Note that the modulo operator can be represented as a linear model [11] and generates a data vector $\mathbf{v}=\tilde{\mathbf{a}}+\mathbf{d} . \mathbf{d}=\left[d_{1}, \ldots, d_{K}\right]^{T}$ is the modulo alphabet which can be eliminated at the receivers by another modulo operator as explicated in Fig. 2.

In optimum case, the spatial CCI approximates to zero $\left(I_{k}^{\prime} \rightarrow 0\right)$. For clarity, we ignore the truncation effect [17] on noise $\tilde{\mathbf{n}}$, and the parallel $K$ streams (8) can be simplified as

$$
\hat{a}_{k}=\tilde{a}_{k}+\tilde{n}_{k}=\sqrt{P_{k}} a_{k}+\tilde{n}_{k}, k=1, \ldots, K .
$$

Note that the transmit power is successfully introduced to each equivalent sub-channel, we may use the TPA scheme to achieve better performance.

\section{TRANSMit POWER Allocation}

We first analyze the precoding loss of MIMO-THP system in section III.A. Followed in section III.B, a TPA scheme is derived by minimizing the BER performance. In the derivations, we assume that ZF-THP is used for transmission, and the derivations of MMSE case are straightforward. Finally, the results of section III.B are applied to an uncoded quadrature amplitude modulation (QAM) in section III.C.

\section{A. Precoding Loss Analysis}

Because of modulo operator in THP, the transmitted symbols $x_{k}$ are assumed to be uniformly distributed, bounded on enlarged range $T_{k}$. As a result, the transmit power will be increasing and is quantified by the precoding loss, which is usually defined for square QAM as $\gamma \triangleq E\left[|x|^{2}\right] / E\left[|\tilde{a}|^{2}\right]=M /(M-1)$ [5], [8], [11]. But in fact, because $\mathbf{B}$ is a unit lower triangular matrix, the first element $\tilde{a}_{1}$ of power loaded data vector $\tilde{\mathbf{a}}$ is directly passed through modulo operator without being interfered by other power loaded data components. Consequently, the distribution of $x_{1}$ is the same with $\tilde{a}_{1}$ rather than the uniform distribution bounded on $T_{1}$. We redefine the precoding loss as

$$
\gamma_{k}=\left\{\begin{array}{l}
1 \quad, k=1 \\
\gamma=M /(M-1), k=2, \ldots, K
\end{array} .\right.
$$

From the analysis above, we note that the precoding loss is negligible for moderate and large modulation sizes, but it should be considered for the small modulation sizes as well. Thus the exact power of $x_{k}$ is given by

$$
\sigma_{x_{k}}^{2}=\gamma_{k} \sigma_{\tilde{a}_{k}}^{2}=\left\{\begin{array}{l}
\sigma_{\tilde{a}_{k}}^{2}=P_{k} \quad, k=1 \\
\gamma \sigma_{\tilde{a}_{k}}^{2}=\gamma P_{k}, k=2, \ldots, K
\end{array},\right.
$$

and the corresponding bound region $t_{K}$ can be calculated as

$$
\frac{t_{k}^{2}}{6}=\sigma_{x_{k}}^{2}=\gamma_{k} P_{k} .
$$

\section{B. Derivation of TPA Scheme}

To derive a TPA scheme, we will express the BER of each transmitted symbol as a function of transmit power $P_{k}$, and find the TPA matrix $\mathbf{P}$ by means of minimizing the average BER. As discussed in section II, from the equivalent model in (10) and the noise covariance matrix (3), the postdetection signal to interference and noise ratio (SINR) $\rho_{k}$ of the $k$-th sub-channel can be expressed as

$$
\rho_{k}=\frac{\sigma_{\tilde{a}_{k}}^{2}}{\sigma_{\tilde{n}_{k}}^{2}}=\frac{P_{k} s_{k k}^{2}}{\sigma_{n}^{2}} .
$$

The average uncoded BER $\bar{P}_{b}$ is then expressed as an arithmetic mean of the BER for every sub-channel

$$
\bar{P}_{b}=\frac{1}{K} \sum_{k=1}^{K} P_{b}\left(\rho_{k}\right),
$$

where $P_{b}\left(\rho_{k}\right)$ is the BER of the $k$-th sub-channel. Hence the TPA scheme that approximately minimizes the average uncoded BER (AMBER-TPA) under sum-power constraint is given by

$$
\begin{aligned}
& \left\{P_{k}\right\}_{k=1}^{K}=\arg \min _{P_{1}, \ldots, P_{K}} \frac{1}{K} \sum_{k=1}^{K} P_{b}\left(\rho_{k}\right) \\
& \text { s.t. } \sum_{k=1}^{K} \gamma_{k} P_{k}=P_{T}
\end{aligned}
$$

where the sum-power constraint is denoted as 


$$
P_{T}=\sum_{k=1}^{K} \sigma_{x_{k}}^{2}=\sum_{k=1}^{K} \gamma_{k} P_{k}=K
$$

We may use the Lagrange multiplier method to deal with this problem in (16) and the cost function may be expressed as

$$
L\left(P_{1}, \ldots, P_{k}, \lambda\right)=\frac{1}{K} \sum_{k=1}^{K} P_{b}\left(\rho_{k}\right)+\lambda\left(\sum_{k=1}^{K} \gamma_{k} P_{k}-K\right) .
$$

From $\partial L / \partial P_{k}=0$, we get a set of $K$ equations

$$
\frac{\partial P_{b}\left(\rho_{k}\right)}{\partial P_{k}}=-\lambda K \gamma_{k}, k=1, \ldots, K
$$

Solving $K+1$ equations in (17) and (19), we may obtain a closed-form expression of TPA matrix $\mathbf{P}$ by minimizing the BER in (15).

\section{Application to Uncoded QAM}

When uncoded $M$-ary QAM is used for THP transmission, the BER for the $K$-th sub-channel can be tightly approximated as an exponential function of $\rho_{k}$ [14], [16]

$$
P_{b}\left(\rho_{k}\right)=\frac{1}{5} \exp \left(-\frac{3 \rho_{k}}{2(M-1)}\right) \text {. }
$$

The average uncoded BER can be expressed as

$$
\bar{P}_{b}=\frac{1}{K} \sum_{k=1}^{K} \frac{1}{5} \exp \left(-\frac{3 \rho_{k}}{2(M-1)}\right)=\sum_{k=1}^{K} \alpha \exp \left(-\beta_{k} P_{k}\right),
$$

where $\alpha=\frac{1}{5 K}$ and $\beta_{k}=\frac{3\left|s_{k k}\right|^{2}}{2(M-1) \sigma_{n}^{2}}$.

Taking (21) into (18) and solving equations (19), the closed-form solution of the transmit power is

$$
P_{k}=\left(\frac{\ln \frac{\alpha \beta_{k}}{\gamma_{k}}-\ln \lambda}{\beta_{k}}\right)^{+}, k=1, \ldots, K,
$$

where $(x)^{+} \triangleq \max (x, 0)$, the Lagrange multiplier $\lambda$ is chosen to satisfy the constraint (17), i.e.,

$$
\lambda=\exp \left(\frac{\sum_{k=1}^{K} \frac{\gamma_{k}}{\beta_{k}} \ln \frac{\alpha \beta_{k}}{\gamma_{k}}-K}{\sum_{k=1}^{K} \frac{\gamma_{k}}{\beta_{k}}}\right) .
$$

Applying (23) to (22), the closed-form expression of TPA matrix is obtained. It should be noted that the TPA scheme in (22) is more reasonable for we considered the precoding loss factor $\gamma_{k}$ and the results are shown in the next section.

\section{SimUlation RESUlTS}

Computer simulations are performed to evaluate the uncoded BER performance over signal to noise ratio (SNR). Two kinds of modulation, square 4QAM and square 16QAM, with Gray encoding are employed [18] during the simulation. We assume $N_{T}=N_{R}=K=4$ i.e., the channel matrix $\mathbf{H}$ is square $4 \times 4$, quasi static and assumed to be perfectly known at the transmitter.

Fig. 3 shows the performance comparisons using square 4QAM modulation for MIMO-THP systems. There are three kinds of curves, which are THP without TPA, conventional approximate minimum SER (AMSER)-TPA [15] and proposed AMBER-TPA (22), employed to THP system under ZF and MMSE case, respectively. It is clearly that both TPA schemes achieve better performance than systems without TPA in all cases. More importantly, because we considered the precoding loss factor, proposed AMBER-TPA also achieves better performance than conventional AMSER-TPA, Under ZF cases, we observe that at BER of $10^{-2}$, proposed AMBER-TPA offers 3.7 and $0.8 \mathrm{~dB}$ SNR gains over THP without TPA and conventional AMSER-TPA schemes, respectively. When MMSE case is employed at BER of $10^{-3}$, proposed AMBERTPA offers nearly 7.4 dB SNR gains over THP without TPA. More importantly, It should be noted that at BER of $10^{-4}$, proposed AMBER-TPA achieves nearly $2.0 \mathrm{~dB}$ SNR gains than conventional AMSER-TPA schemes.

Fig.4 shows the performance comparisons using square 16QAM modulation for MIMO-THP systems. There are also six curves the same as Fig. 3. Noteworthy, both under ZF case and MMSE case, the curves of proposed AMBER-TPA and AMSER-TPA are almost indistinguishable. This indicates that when the modulation size is moderate, the precoding loss effect of TPA is negligible. Because the AMSER-TPA scheme in [15] needs iterative procedure, and yet the proposed AMBER-TPA can get a closed-form solution, complexity of the latter algorithm is lower. Therefore, we focus on the comparisons between proposed AMBER-TPA and THP without TPA. At BER of $10^{-2}$, the ZF-THP with AMBER-TPA gives approximately $2.4 \mathrm{~dB}$ gains over ZF-THP without TPA, and the MMSE-THP with AMBER-TPA gives approximately $2.1 \mathrm{~dB}$ gains over MMSE-THP without TPA.

\section{CONCLUSIONS}

An AMBER-TPA algorithm for MIMO-THP system is proposed under flat fading scenarios. This scheme is achieved by minimizing the average uncoded BER under sum-power constraint and considering the precoding loss. The comparisons show that the proposed AMBER-TPA method can effectively improve the BER performance of MIMO-THP systems without TPA. Furthermore, it is not only more efficient calculation, but also achieves better performance than AMSER-TPA method in [15]. Finally, above results are derived under perfect conditions, while in fact it is difficult to obtain ideal information. An important topic for our future work is to apply the algorithm to more realistic situations, such as extended to multiuser case or coded MIMO system and analysis of the penalty for using imperfect or outdated channel information. 


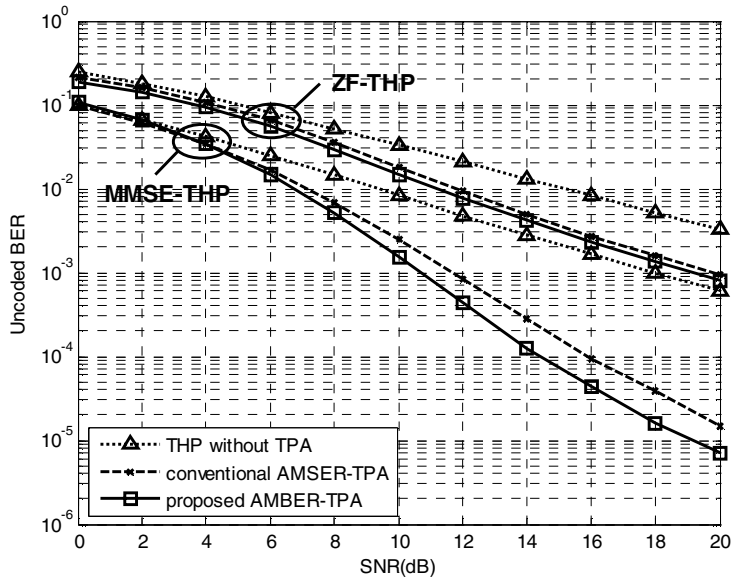

Figure 3. Uncoded BER performance comparisons of proposed AMBERTPA, conventional AMSER-TPA and without TPA in MIMO-ZF/MMSETHP for square 4QAM ( $\left.N_{T}=N_{R}=4\right)$.

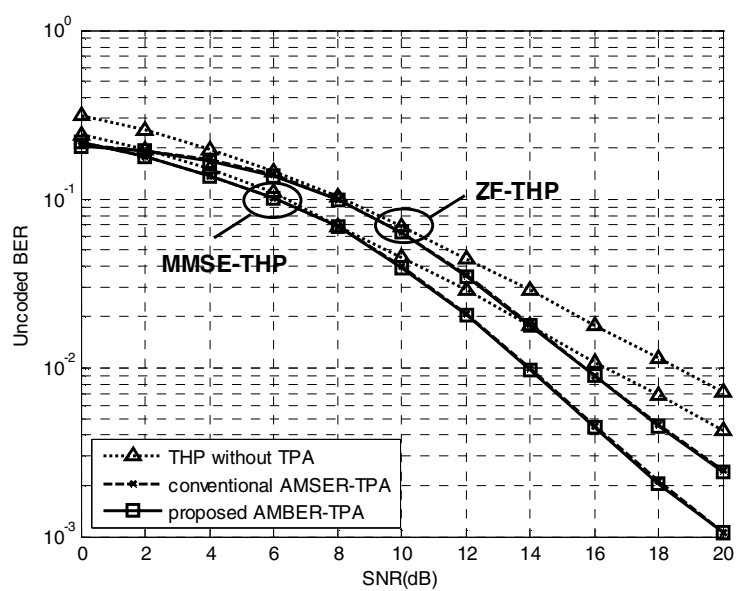

Figure 4. Uncoded BER performance comparisons of proposed AMBERTPA, conventional AMSER-TPA and without TPA in MIMO-ZF/MMSE-

THP for square 16QAM ( $\left.N_{T}=N_{R}=4\right)$.

\section{ACKNOWLEDGMENT}

The authors would like to thank the editors and anonymous reviewers for their helpful comments.

\section{REFERENCES}

[1] G. J. Foschini and M. J. Gans, "On limits of wireless communications in a fading environment when using multiple antennas," Wireless Pers. Commun., vol. 6, pp. 315-335, Mar. 1998.

[2] E. Telatar. Capacity of multi-antenna Gaussian channels. European Transactions on Telecommunications, pp. 585-596, Nov. 1999.

[3] P. W. Wolniansky, G. J. Foschini, G. D. Golden, and R. A. Valenzuela, "V-BLAST: An architecture for realizing very high data rates over the rich-scattering wireless channel," presented at the ISSSE-98, Pise, Italy, Sept. 1998. invited paper.

[4] G. Ginis and J. M. Cioffi, "On the relation between V-BLAST and the GDFE,” IEEE Commun. Lett., pp. 364-366, Sept. 2001.

[5] R.F.H. Fischer, C. Windpassinger, A. Lampe, and J.B. Huber, "SpaceTime Transmission using Tomlinson-Harashima Precoding," In Proc. of 4. ITG Conf. on Source and Channel Coding, pp. 139-147, January 2002.

[6] M. Tomlinson, "New automatic equaliser employing modulo arithmetic," Electronics Letters, pp. 138-139, March 1971.

[7] H. Harashima and H. Miyakawa, "Matched-transmission technique for channels with intersymbol interference," IEEE Trans. Commun., vol. COM-20, pp. 774-780, Aug. 1972.

[8] P. Edward; L. Ying-Chang, "Power and Modulo Loss Tradeoff for Tomlinson-Harashima Precoding Applied to Geometric Mean Decomposition based MIMO Systems," In Proc. of 18th. PIMRC, pp. 15, Sep. 2007

[9] A. J. Goldsmith and P. P. Varaiya, "Capacity of fading channels with channel side information," IEEE Trans. Inf. Theory, vol. 43, pp. 19861992,Nov. 1997.

[10] G. Caire, G. Taricco, and E. Biglieri, "Optimum power control over fading channels," IEEE Trans. Inf. Theory, vol. 45, pp. 1468-1489, July 1999.

[11] C. Windpassinger, R. F. H. Fisher, T. Vencel, and J. B. Huber, "Precoding in multiantenna and multiuser communications," IEEE Trans. Wireless Commun., vol. 3, no. 4, pp. 1305-1316, July 2004.

[12] M. Payar'o, A. P'erez-Neira, and M. Lagunas, "Achievable rates for generalized spatial Tomlinson-Harashima precoding in MIMO systems," in Proc. IEEE Vehic. Tech. Conf. Fall, Sept. 2004.

[13] S. H. Nam, Oh-S. Shin, K. B. Lee, "Transmit power allocation for a modified V-BLAST system," IEEE Trans. Commun., vol. 52, pp. 1074 1079, July 2004.

[14] N. Wang, S. D. Blostein, "Minimum BER power allocation for MIMO spatial multiplexing systems," IEEE Trans. Commun., vol. 55, pp. 180 187, Jan. 2007.

[15] Bizaki, H.K. and Falahati, A, "Power Loading by Minimizing the Average Symbol Error Rate on MIMO THP Systems," In Proc. of 9. ICACT. Vol. 2, pp. 1323-1326, Feb. 2007.

[16] S. Zhou and G. B. Giannakis, "Adaptive modulation for multi-antenna transmissions with channel mean feedback," in Proc. IEEE ICC, Anchorage, AK, vol. 4, pp. 2281-2285, May 2003.

[17] Richard D. Wesel, and John M. Cioffi, "Achievable Rates for Tomlinson-Harashima Precoding" IEEE Trans. on Inf. Theory, Vol.44 No.2, March 1998

[18] J. G. Proakis "Digital communications (4th edition)," New York, NY:McGraw-Hill, 2001. 